\title{
Phase relationships between velocity, wall pressure, and wall shear stress in a forced turbulent boundary layer
}

\author{
Kevin Rosenberg*, Subrahmanyam Duvvuri ${ }^{\dagger}$, Mitul Luhar ${ }^{\ddagger}$, Beverley J. McKeon ${ }^{\S}$ \\ California Institute of Technology, Pasadena, California, 91125 \\ Casey Barnard ^, Brett Freidkes ॥, Jessica Meloy **, Mark Sheplak ${ }^{\dagger \dagger}$ \\ University of Florida, Gainesville, Florida, 32611
}

\begin{abstract}
A large scale spatio-temporally periodic disturbance was excited in a turbulent boundary layer via a wall-actuated dynamic roughness. Streamwise velocity, wall pressure, and direct wall shear stress measurements were made with a hot wire, pressure microphone, and a micro-scale differential capacitive sensor, respectively. Phase-averaged fields for the three quantities were calculated and analyzed. A phase calibration between the various sensors was performed with an acoustic plane wave tube over a range of operating conditions to validate a direct phase comparison between the respective quantities. Results suggest encouraging agreement between the phase of the wall shear stress and velocity near the wall; however, more refined velocity measurements are needed to make quantitative comparisons to the wall pressure. Overall, this work highlights the potential for wall-based control with applications towards reducing turbulent drag.
\end{abstract}

\section{Nomenclature}

\begin{tabular}{ll}
$U_{\infty}$ & Free stream velocity \\
$\delta$ & Boundary layer thickness \\
$\nu$ & Kinematic viscosity \\
$\mu$ & Dynamic viscosity \\
$\rho$ & Density \\
$c$ & Isentropic speed of sound \\
$\eta$ & Stokes number \\
$k_{x}$ & Streamwise wavenumber \\
$u$ & Streamwise velocity \\
$\tau_{w}$ & Wall shear stress \\
$p_{w}$ & Wall pressure \\
$p^{+}$ & Incident pressure phasor \\
$p^{-}$ & Reflected pressure phasor \\
$x$ & Axial location within plane wave tube \\
$y$ & Transverse location within plane wave tube \\
$\widetilde{()}$ & Phase-averaged quantity \\
CSSS & Capacitive shear-stress sensor \\
\hline
\end{tabular}

\footnotetext{
*PhD Candidate, Graduate Aerospace Laboratories, AIAA Student Member.

${ }^{\dagger}$ Postdoctoral Scholar, Graduate Aerospace Laboratories, AIAA Student Member.

$\ddagger$ Current Affiliation: Assistant Professor, Aerospace \& Mechanical Engineering, USC, AIAA Member.

$\S$ Professor of Aeronautics, Graduate Aerospace Laboratories, AIAA Associate Fellow.

IPhD Candidate, Mechanical \& Aerospace Engineering, AIAA Student Member.

" PhD Student, Mechanical \& Aerospace Engineering

** Current Affiliation: Engineer, Boeing Test \& Evaluation, AIAA Member.

${ }^{\dagger}$ Professor, Mechanical \& Aerospace Engineering, Computer \& Electrical Engineering, AIAA Associate Fellow.
} 


$\begin{array}{ll}\text { PWT } & \text { Plane wave tube } \\ \text { R } & \text { Pressure reflection coefficient } \\ \text { TMM } & \text { Two-microphone method } \\ \text { TBL } & \text { Turbulent boundary layer }\end{array}$

\section{Introduction}

Turbulent drag reduction continues to be an important engineering challenge, and the potential economic and environmental impact is well-documented. ${ }^{1}$ This study seeks to further the viability of practical wall-based control by experimentally manipulating a turbulent boundary layer and analyzing the measured velocity and wall-sensed fields. Previous studies ${ }^{2,3}$ have demonstrated the efficacy of using a wall-actuated dynamic roughness to excite a single coherent spatio-temporal mode in a turbulent boundary layer. This study furthers this previous work, notably by investigating the agreement between experiment and theory for the phase behavior between the velocity and the wall-sensed quantities, i.e., the wall pressure and wall shear stress. Time-resolved wall shear stress measurements are vital for this investigation, and this study uses a novel micro-scale differential capacitive sensor to make these measurements. The capacitive wall shear stress sensor ${ }^{4,5}$ (CSSS) is a bulk silicon micro-machined floating element device with H-bar tether structure. Interdigitated comb fingers act as variable gap capacitors, with a synchronous modulation/demodulation interface circuit providing real time signal response for mean and fluctuating inputs.

\section{Experimental Setup}

Since the goal of this study is to directly compare the phase behavior in a turbulent boundary layer between three flow quantities obtained by three different sensors, a phase calibration is necessary to validate these comparisons. For the calibration, an acoustic plane wave tube (PWT) with a square cross section of 8.5 $\mathrm{x} 8.5 \mathrm{~mm}^{2}$ is designed to produce plane waves up to $20 \mathrm{kHz} .{ }^{6}$ For this procedure, sinusoidal signals between $300 \mathrm{~Hz}$ and $2000 \mathrm{~Hz}$ are examined. The PWT is modified to allow the insertion of a Dantec boundary layer hot-wire probe (type 55P14) that measures velocity, two $3.18 \mathrm{~mm}\left(1 / 8^{\prime \prime}\right)$ Brüel \& Kjær pressure-field microphones (type 4138), and a capacitive wall shear stress sensor. The CSSS has a sensing area of $1 \mathrm{~mm}$ x $1 \mathrm{~mm}$, a sensitivity of $4.52 \mathrm{mV} / \mathrm{Pa}$ at $1.128 \mathrm{kHz}$, a minimum detectable shear of $340 \mu \mathrm{Pa}$ at $50 \mathrm{~Hz}$, and a resonant frequency of $2.7 \mathrm{kHz}$. A JBL $2426 \mathrm{H}$ compression driver produces the plane waves at the inlet of the $1.45 \mathrm{~m}$ long tube. The inlet also has a junction which allows for mean air flow at approximately $4 \mathrm{~m} / \mathrm{s}$ (maintained by FMA-2611A flow controller) to pass through the tube, which is necessary to avoid hot-wire signal rectification. The termination of the PWT, which determines the reflected behavior of the incident acoustic waves, is designed accordingly to provide an outlet for the air and to minimize reflections caused by acoustic impedance mismatches. This is accomplished via an $0.46 \mathrm{~m}$ long extension of the PWT that feeds into a $15 \mathrm{~m}$ long, $12.7 \mathrm{~mm}$ diameter Tygon plastic tubing. These additions provide viscous dissipation that dampens reflected acoustic energy. The hot wire (nominally placed at the channel centerline), one of the microphones, and the CSSS are all placed at the same axial location $5.08 \mathrm{~cm}$ away from the extension. A second microphone required for measuring the reflection coefficient is $2.54 \mathrm{~cm}$ further upstream. The experimental setup is shown in figure 1.

Assuming small, linear perturbations, analytical expressions ${ }^{6}$ can be obtained for the pressure, velocity, and wall shear stress fluctuations in the tube and thus provide a means to determine a theoretical phase relationship between the various quantities. For the general case of incident and reflected waves, these expressions take the following form,

$$
\begin{gathered}
p=p^{+} e^{i\left(\omega t+k_{x} x\right)}+p^{-} e^{i\left(\omega t-k_{x} x\right)}=p^{+}\left[e^{i\left(\omega t+k_{x} x\right)}+R e^{i\left(\omega t-k_{x} x\right)}\right] \\
u=-\frac{p^{+}}{\rho c}\left(1-\frac{\cosh \left(\frac{y}{b} \eta \sqrt{i}\right)}{\cosh (\eta \sqrt{i})}\right)\left[e^{i\left(\omega t+k_{x} x\right)}-R e^{i\left(\omega t-k_{x} x\right)}\right] \\
\tau_{w}=p^{+} \frac{\sqrt{i \nu \omega}}{c} \tanh (\eta \sqrt{i})\left[e^{i\left(\omega t+k_{x} x\right)}-R^{i\left(\omega t-k_{x} x\right)}\right]
\end{gathered}
$$




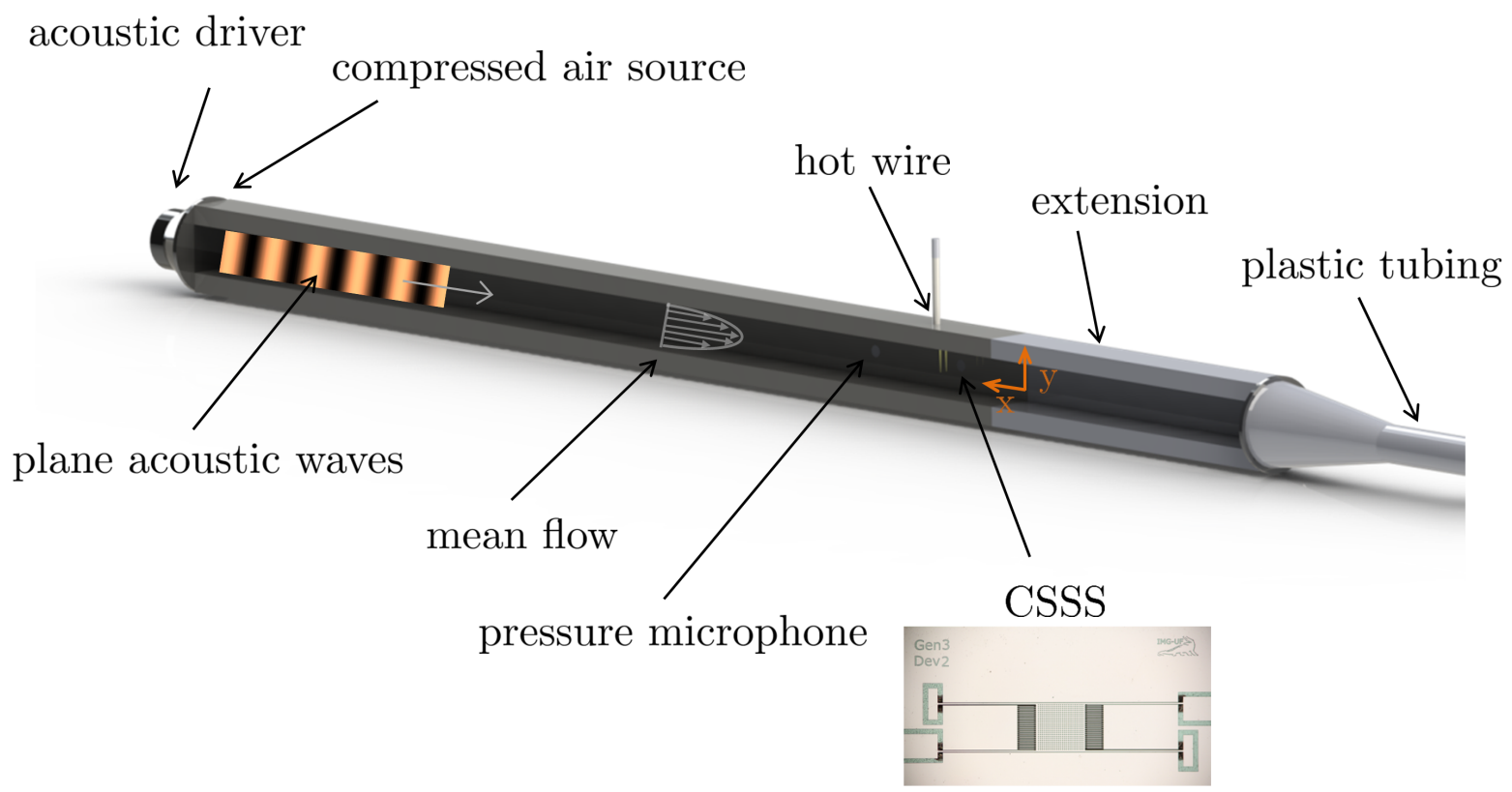

Figure 1. Schematic of PWT used for phase calibration measurements. The side wall is removed for visualization purposes, though it should be noted a second pressure microphone is mounted on this wall at the same axial location as the hot wire and CSSS

where $p, u$, and $\tau_{w}$ are the pressure, velocity, and wall shear stress measured a distance $x$ from the extension, $p^{+}$and $p^{-}$are the incident and reflected pressure phasors ${ }^{7}$ at the extension junction, respectively, $k_{x}=\frac{\omega}{c}$ is the streamwise wavenumber, $b$ is the channel half-height, $y$ is the transverse position in the tube relative to the centerline, $\eta=\sqrt{\frac{\omega b^{2}}{\nu}}$ is the Stokes number, $i=\sqrt{-1}$, and $\mathrm{R}$ is the pressure reflection coefficient. $\mathrm{R}$ is experimentally determined using the two-microphone method $^{8}$ (TMM),

$$
R=\left[\frac{H_{12}(\omega)-e^{-i k_{x} s}}{e^{i k_{x} s}-H_{12}(\omega)}\right] e^{i 2 k_{x}(s+l)}
$$

where $H_{12}(\omega)$ is the measured transfer function between the two microphones, $s$ is the separation distance between the microphones, and $l$ is the distance from the extension to the first microphone.

Knowledge of $\mathrm{R}$ and the driving frequency of the speaker allows for the determination of the analytical phase difference between the aforementioned quantities using equations 1,2 , and 3 . These relations are complex, meaning that the analytical phase angle between the two quantities is found by evaluating the arctangent of the ratio of the imaginary component to the real component. Analytically, the wall shear stress response always leads the velocity response by 45 degrees and is independent of the reflection coefficient.

Experimental data for the calibration procedure is collected using a National Instruments PXI-4498 data acquisition module on an NI PXI-1042Q chassis. Data from the hot wire, microphones, and CSSS are sampled at $200 \mathrm{kHz}$ for 25 seconds. The experimental phase values between different measurements are found in two ways. First, a transfer function is estimated between the wall shear stress and the velocity voltage sets using the MATLAB function tfestimate. The phase between the two measurements is found at the driving frequency of the speaker. Second, the phase between the CSSS and the hot-wire is found by using the microphone as an intermediary reference. Using the MATLAB function xcorr, the cross correlation between the microphone and the CSSS voltage data is determined. An experimental phase is found using the sampling frequency, acoustic frequency, and the calculated cross correlation. Then, the phase difference between the hot-wire and the microphone data is estimated using the same procedure. Comparing the responses ultimately yields an experimental phase estimate between the CSSS and the hot-wire. 
After the phase calibration was performed, the sensors were mounted in the Merrill wind tunnel at the California Institute of Technology. The tunnel has an adjustable height ceiling allowing for a nominally zero-pressure-gradient boundary layer to develop over a flat plate. As shown in figure 2, the flat plate has an insert to allow for a thin, spanwise aligned rib to oscillate up and down at a prescribed frequency. This setup was previously used by Duvvuri \& McKeon, ${ }^{9}$ where additional details can be found. At the trough of the oscillation the rib sits flush with the wall and the peak-to-peak amplitude is set by the user. The rib is driven by a linear actuator (Bose, LM1) and its position is recorded with a linear encoder to allow for phaselocked measurements. The dynamic roughness introduces a nominally spanwise-constant disturbance with a dominant time-scale and a dominant streamwise wavelength into the flow, characterized by the wavenumberfrequency pair $\left(k_{x}, k_{z} \approx 0, \omega\right)$, whose presence is detected in the downstream sensors. The CSSS and the microphone are mounted flush at the wall (not simultaneously) at the same streamwise location as the hotwire which corresponded to a distance of approximately $5 \delta$ downstream of the roughness, where $\delta$ is the local boundary layer thickness. A full traverse of the hot-wire is performed to record velocity across the thickness of the boundary layer. Data was recorded with an NI-DAQ 6220 at a sampling frequency of $60 \mathrm{kHz}$ and a record length of $80 \mathrm{~s}$. For these particular set of experiments, the free stream velocity was $U_{\infty}=22.4 \mathrm{~m} / \mathrm{s}$ yielding a boundary layer thickness of approximately $\delta \approx 17 \mathrm{~mm}$, a Reynolds number based on momentum thickness of $R e_{\theta} \approx 3100$, and a friction Reynolds number of $R e_{\tau} \approx 990$. The active sensing length for the hot wire, microphone, and CSSS normalized in viscous wall units is 70, 180, and 56, respectively. The roughness frequency and amplitude was $50 \mathrm{~Hz}$ and $1 \mathrm{~mm}$ respectively. This frequency was chosen to allow for comparison to previous studies ${ }^{3,9}$ which have thoroughly characterized similarly scaled disturbances. The corresponding streamwise wavenumber of the disturbance is estimated by tracking the change in phase of the synthetic mode with streamwise distance ${ }^{3}$ and is found to be $k_{x} \approx \frac{2 \pi}{15 \delta}$.

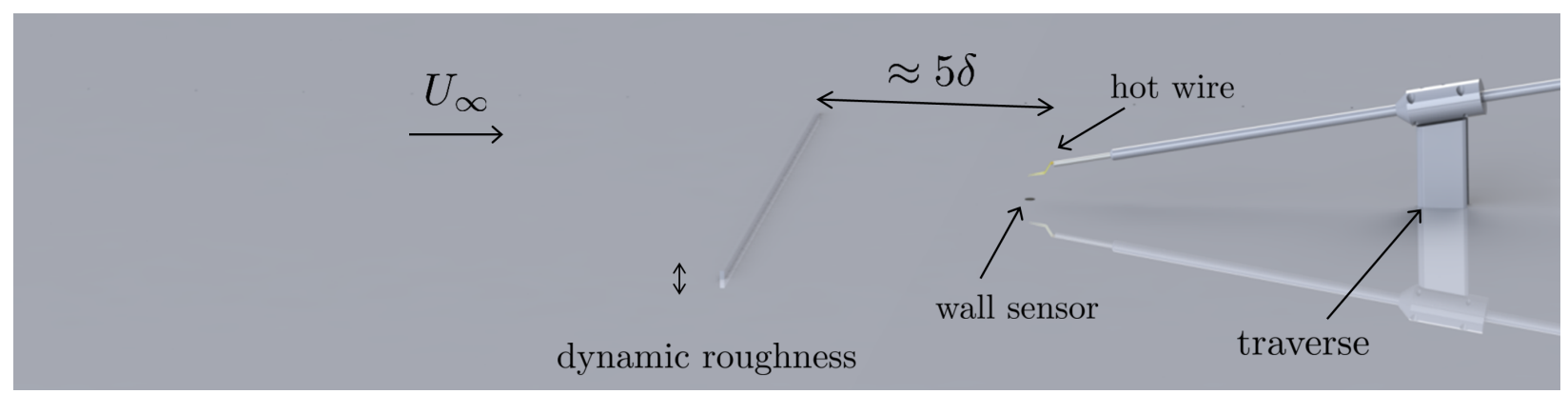

Figure 2. Flat plate boundary layer with dynamic roughness and location of downstream sensors

\section{Results}

First, we highlight the main results from the PWT measurements, with a brief discussion of uncertainty. Then, we present and analyze the results from the TBL measurements.

\section{A. PWT measurements}

A subset of results from measurements in the PWT over a range of frequencies is found in Table 1 . The lowest frequency reported corresponds to the operating limit of the compression driver. Experimentally measured phase differences between the various quantities are reported along with their corresponding theoretical values. The results suggest a phase lag between the CSSS and hot wire as high as approximately 20 degrees, though it appears less pronounced at lower frequencies. The phase lag between the microphone and CSSS is slightly lower, with a value of approximately 10 degrees, and less of a frequency dependence. A Monte Carlo uncertainty analysis was performed to determine the error associated in the analytical phases calculated. Uncertainty exists due to errors that arise from PWT machining, calculation of the reflection coefficient, air properties such as speed of sound and kinematic viscosity, and acquisition errors associated with the sensitivities of the different transducers. The analysis revealed theoretical uncertainties on the order of 5 degrees, which places many of the phase measurements outside this bound. This suggests the existence of a 
non-negligible phase lag between the various sensors. It is important to note that the theoretical uncertainty value did not reflect any inherent phase uncertainty in the instrumentation; thus, there may be a connection between the observed phase lag and the instrumentation itself. Future studies will seek to further quantify this connection and the behavior of the phase lags, particularly for frequencies closer to the desired $50 \mathrm{~Hz}$ roughness frequency.

\begin{tabular}{|c|c|c|c|}
\hline Frequency $(\mathrm{Hz})$ & $|R|$ & $\theta_{\tau_{w}}-\theta_{u}$ & $\theta_{p_{w}}-\theta_{\tau_{w}}$ \\
\hline 300 & 0.2056 & $45.44(45)$ & $120.60(121)$ \\
\hline 700 & 0.2219 & $49.75(45)$ & $122.94(141)$ \\
\hline 1100 & 0.2125 & $66.39(45)$ & $139.14(153)$ \\
\hline 1500 & 0.2255 & $61.14(45)$ & $154.80(161)$ \\
\hline 1900 & 0.2197 & $61.78(45)$ & $151.92(155)$ \\
\hline
\end{tabular}

Table 1. Experimentally measured phase differences in the PWT over a range of frequencies with theoretical values in parentheses. In this notation, shear leads velocity and pressure leads shear.

\section{B. TBL measurements}

Following the procedure of Hussain \& Reynolds, ${ }^{10}$ the velocity signal is decomposed as

$$
u=\bar{U}+\widetilde{u}+u^{\prime}
$$

where $\bar{U}$ is the time-averaged mean, $\widetilde{u}$ is the spatio-temporally periodic component excited by the dynamic roughness, and $u^{\prime}$ is the remaining turbulent fluctuations. Using the encoder signal of the roughness as a phase reference, the mean-subtracted velocity signal is phase-averaged with respect to the $50 \mathrm{~Hz}$ input to extract $\widetilde{u}$. Similarly, this procedure is applied to the wall shear stress and wall pressure signals to obtain $\widetilde{\tau}_{w}$ and $\widetilde{p}_{w}$. The phase map for a single period of $\widetilde{u}(y), \widetilde{\tau}_{w}$, and $\widetilde{p}_{w}$, without phase corrections, is shown in figure 3 . It is seen near the wall that $\widetilde{u}$ leads $\widetilde{\tau}_{w}$ by approximately 20 degrees, and $\widetilde{p}_{w}$ leads $\widetilde{\tau}_{w}$ by approximately 140 degrees.

The theoretical phase relationship between the three quantities near the wall can be estimated by considering a Taylor series expansion of the velocity about the wall.

$$
\left.\widetilde{u}(y) \approx \widetilde{u}\right|_{y=0}+\left.\frac{\partial \widetilde{u}}{\partial y}\right|_{y=0} y+\left.\frac{\partial^{2} \widetilde{u}}{\partial y^{2}}\right|_{y=0} y^{2}+\mathcal{O}\left(y^{3}\right)+\ldots
$$

By definition, we have

$$
\left.\frac{\partial \widetilde{u}}{\partial y}\right|_{y=0}=\frac{1}{\mu} \widetilde{\tau}_{w}
$$

and by evaluating the $\mathrm{u}$-momentum equation at the wall it can be shown that

$$
\left.\frac{\partial^{2} \widetilde{u}}{\partial y^{2}}\right|_{y=0}=\frac{1}{\mu} \frac{\partial \widetilde{p}_{w}}{\partial x}=\frac{i k_{x}}{\mu} \widetilde{p}_{w}
$$

From (6),(7) and the no-slip condition we have

$$
\widetilde{u}(y) \approx \frac{1}{\mu} \widetilde{\tau}_{w} y+\frac{i k_{x}}{\mu} \widetilde{p}_{w} y^{2}+\mathcal{O}\left(y^{3}\right)+\ldots
$$

Thus, very near the wall we expect $\widetilde{u}$ and $\widetilde{\tau}_{w}$ to be in phase, which is observed in figure 3 . By expressing the phase-averaged quantities in polar form, $\widetilde{()}=|\widetilde{()}| e^{i \theta_{(}}$it can be shown via Taylor series expansion that 

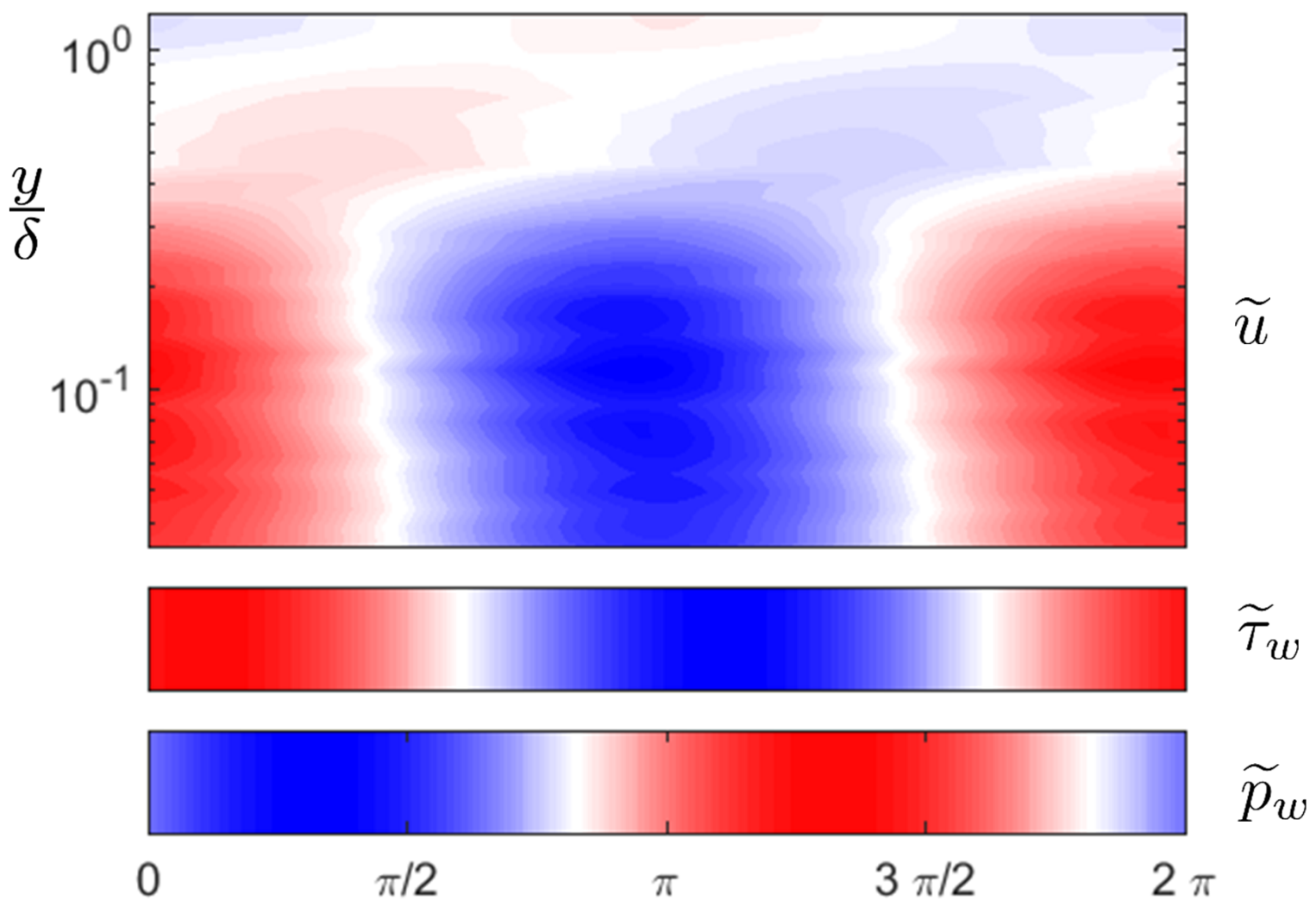

Figure 3. Phase-averaged velocity $\widetilde{u}$ (top), wall shear stress $\widetilde{\tau}_{w}$ (middle) and wall pressure $\widetilde{p}_{w}$ (bottom) signals for the $50 \mathrm{~Hz}$ mode over a single period. These represent the measured values, no phase correction has been applied. Note: the color in the bottom panels is intended to highlight phase and does not imply equal magnitude

$$
\left.\frac{\partial \theta_{u}}{\partial \frac{y}{\delta}}\right|_{\frac{y}{\delta}=0} \approx-\frac{\left|\widetilde{p}_{w}\right|}{\left|\widetilde{\tau}_{w}\right|} \cos \left(\theta_{p_{w}}-\theta_{\tau_{w}}\right) k_{x} \delta
$$

Therefore, establishing a theoretical phase relationship with $\theta_{p_{w}}$ requires an accurate estimate of the wallnormal gradient of $\theta_{u}$, which would ideally require velocity measurements within the viscous sublayer. For this study, the nearest velocity measurement was made at a $y^{+} \approx 34$ so estimating this gradient is currently not feasible. Based on the experimentally measured phase difference between $\widetilde{p}_{w}$ and $\widetilde{\tau}_{w}$ the value of $\frac{\partial \theta_{u}}{\partial \frac{y}{\delta}}$ is positive indicating $\widetilde{u}$ would lead $\widetilde{\tau}_{w}$ near the wall, which is in qualitative agreement with the results in figure 3. Future work will investigate using high-speed PIV to obtain accurate near-wall velocity measurements to allow for more quantitative comparisons, particularly to the estimated lags from the PWT calibration.

\section{Summary \& Conclusions}

Experiments were performed to characterize the phase behavior between velocity, wall pressure, and wall shear stress in a TBL in the presence of an excited spatio-temporal mode. A phase calibration between the various sensors was performed in a PWT. Results suggest instrumentation phase lags on the order of 10-20 degrees in the context of a direct phase comparison between the different sensor outputs. Future investigations will seek to further quantify the frequency dependence of the lags, particularly at lower frequencies. The measured phase values in the TBL are encouraging, though improved near-wall velocity measurements are needed for a more thorough comparison. Future studies will also explore the agreement between experimental measurements and predictions from the resolvent model of McKeon \& Sharma. ${ }^{11}$ The resolvent 
model is particularly well-suited for this approach since it allows for the analysis of the behavior of individual spatio-temporal modes. Characterizing the phase behavior for these various quantities within the context of the deterministic resolvent model would be an important step towards implementing wall-based feedback control of turbulent flows.

\section{Acknowledgments}

The support of AFOSR (grant FA 9550-12-1-0469, program manager D. Smith) is gratefully acknowledged.

\section{References}

${ }^{1}$ Alonso J, Balakrishnan H, Kroo J, and Tomlin C, Energy-efficient air transportation, in The Impact of Control Technology, edited by Samad, T and Annaswamy,A.M. (IEEE Control Systems Society, 2011).

${ }^{2}$ Jacobi, I. and McKeon, B. J. (2011) Dynamic roughness perturbation of a turbulent boundary layer. Journal of Fluid Mechanics, 688, pp. 258-296.

${ }^{3}$ Duvvuri, S. and McKeon, B.J. "Phase Relationships in Presence of a Synthetic Large-Scale in a Turbulent Boundary Layer", 44th AIAA Fluid Dynamics Conference, AIAA Aviation, (AIAA 2014-2883).

${ }^{4}$ Chandrasekharan V, Sells J, Meloy JC, Arnold DP, Sheplak M (2011) A Microscale Differential Capacitive Direct WallShear-Stress Sensor. Journal of Microelectromechanical Systems 20(3):622635

${ }^{5}$ Meloy J, Griffin J, Sells J, Chandrasekharan V, Cattafesta LN, Sheplak M (2011) Experimental Verification of a MEMS Based Skin Friction Sensor for Quantitative Wall Shear Stress Measurement. In: 41st AIAA Fluid Dynamics Conference, AIAA, Honolulu, HI, June, pp 118. (AIAA 2011-3995).

${ }^{6}$ Chandrasekaran, V., Cain, A., Nishida, T., Cattafesta, L. N., \& Sheplak, M. (2005). Dynamic calibration technique for thermal shear-stress sensors with mean flow. Experiments in Fluids, 39(1), 56-65.

${ }^{7}$ Blackstock, D.T. Fundamentals of physical acoustics." New York, NY: Wiley, 2000.

${ }^{8}$ Jones MG and Stiede PE (1997) Comparison of methods for determining specific acoustic impedance. J Acoust Soc Am 101(5):26942704

${ }^{9}$ Duvvuri, S. and McKeon, B. J. (2015) Triadic scale interactions in a turbulent boundary layer. Journal of Fluid Mechanics, 767 .

${ }^{10}$ Hussain, A. K. M. F. and Reynolds, W.C., The mechanics of an organized wave in turbulent shear flow, Journal of Fluid Mechanics(1970), vol. 41-2, ]pp. 241-258.

${ }^{11}$ McKeon, B. J. and Sharma, A. S. (2010) A critical-layer framework for turbulent pipe flow. Journal of Fluid Mechanics, 658, pp. 336-382 